\title{
Massive Spontaneous Haemothorax after Rivaroxaban Therapy for Acute Pulmonary Embolism
}

\author{
Denise Tan Yan ${ }^{1,2}$, Raymond Goh Kai Heng ${ }^{1}$, Heng Joo Ng² \\ ${ }^{1}$ Department of Medicine, Sengkang General Hospital, Singhealth, Singapore \\ ${ }^{2}$ Department of Haematology, Singapore General Hospital, Singhealth, Singapore
}

Received: 26/07/2019

Accepted: 31/07/2019

Published: 18/09/2019

How to cite this article: Tan YD, Goh KHR, Ng HJ. Massive spontaneous haemothorax after rivaroxaban therapy for acute pulmonary embolism. EJCRIM 2019;6: doi:10.12890/2019_001236.

Conflicts of Interests: The Authors declare that there are no competing interest

This article is licensed under a Commons Attribution Non-Commercial 4.0 License

\section{ABSTRACT}

Spontaneous haemothorax complicating the treatment of pulmonary embolism is rare and potentially fatal. We describe a patient with pulmonary embolism and severe pleuritic pain who developed a life-threatening haemothorax 10 days later while on rivaroxaban therapy. This case highlights the fact that severe pleuritic pain associated with pulmonary embolism may indicate subclinical infarction of tissue near the visceral pleura with an increased risk of pleural effusion and the subsequent development of a haemothorax. It is important to recognise such danger signs warranting closer attention, especially since the increased use of direct oral anticoagulants has facilitated ambulatory care and this complication may manifest in the outpatient setting.

\section{LEARNING POINTS}

- Spontaneous haemothorax may occur in the first 2 weeks after a patient starts anticoagulation.

- Severe pleuritic pain in a patient with pulmonary embolism may indicate subclinical infarction near the visceral pleura with an increased risk of pleural effusion and the subsequent development of a spontaneous haemothorax.

- Patients with severe pain and pleural effusion should be monitored closely, especially if they are outpatients, even though initial radiological findings are not significant.

\section{KEYWORDS}

Spontaneous haemothorax, pulmonary embolism, pulmonary infarction, rivaroxaban, anticoagulation

\section{INTRODUCTION}

Massive spontaneous haemothorax complicating the treatment of pulmonary embolism is rare and potentially fatal. It is caused by the rupture of small blood vessels, often in the presence of a pulmonary infarct ${ }^{[1]}$. Severe pleuritic pain, especially with associated pleural effusion, suggests subpleural ischaemia or infarction which may not always be radiologically detectable.

Here we describe a patient with pulmonary embolism and severe pleuritic pain who subsequently developed a life-threatening spontaneous haemothorax on rivaroxaban therapy.

\section{CASE DESCRIPTION}

A 24-year old man presented with a 1-day history of severe left-sided pleuritic chest pain after 8 weeks of immobilisation of his right foot for a metatarsal fracture. His was febrile with a temperature of $38.6^{\circ} \mathrm{C}$ but vital signs were stable. He had no significant medical or bleeding history. 
His blood counts, coagulation profile and chest x-ray (CXR) were normal (Fig. 1). A computed tomography pulmonary angiogram (CTPA) showed pulmonary embolism involving bilateral lower lobe segmental arteries and their branches with a small left-sided pleural effusion, but without the typical features of a pulmonary infarct (Fig. 2).

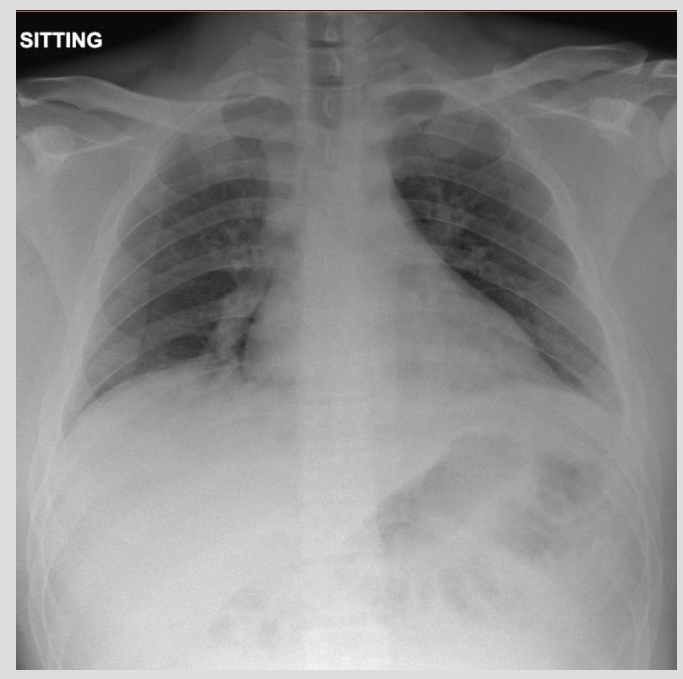

Figure 1. Chest $x$-ray at first presentation

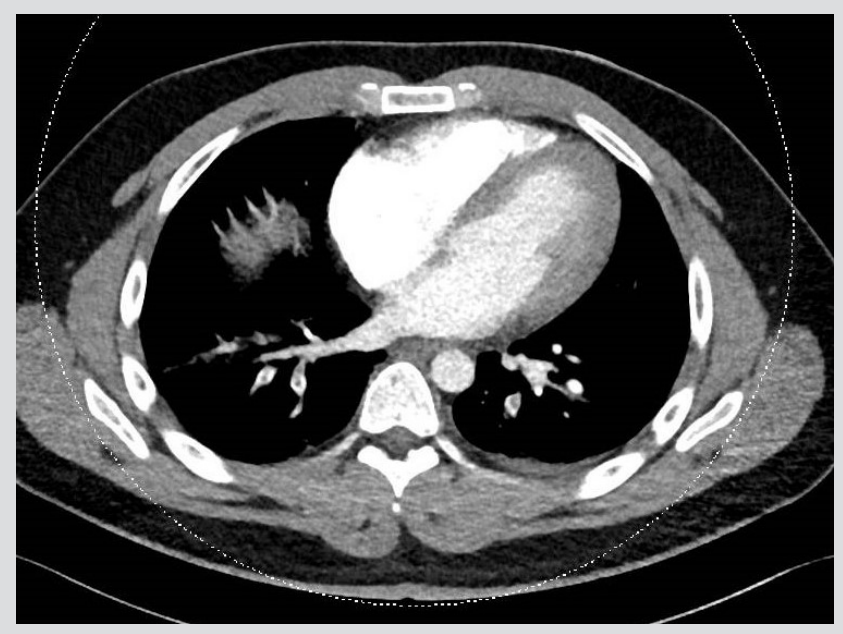

Figure 2. CT pulmonary angiogram showing bilateral lower lobe segmental artery thrombosis

Enoxaparin at $1 \mathrm{mg} / \mathrm{kg}$ administered every 12 hours was initiated. It was unusual that his pain was out of proportion to the radiological findings and was difficult to control, even with regular morphine. He was clinically monitored for 5 more days until his pain improved, then discharged with oral opioids and rivaroxaban $15 \mathrm{mg}$ twice daily.

The patient returned 5 days later with worsening pleuritic chest pain and dyspnoea that began 2 days after discharge. A CXR now showed a moderate-sized left pleural effusion (Fig. 3). His vital signs were initially normal but he developed haemodynamic shock within 12 hours of presentation. Urgent CXR after fluid resuscitation showed progression into a massive effusion with mediastinal shift (Fig. 4). CTPA did not show any new pulmonary embolisms but confirmed a large pleural effusion with dependent hyperdense contents suspicious for haemorrhage and passive collapse of the left lung with a rightward mediastinal shift (Fig. 5).

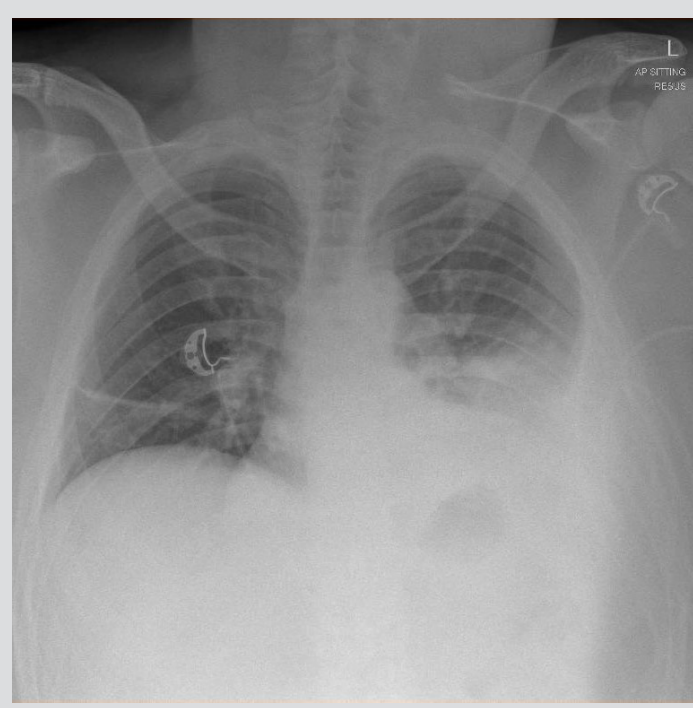

Figure 3. Chest $x$-ray showing a moderate-sized left pleural effusion

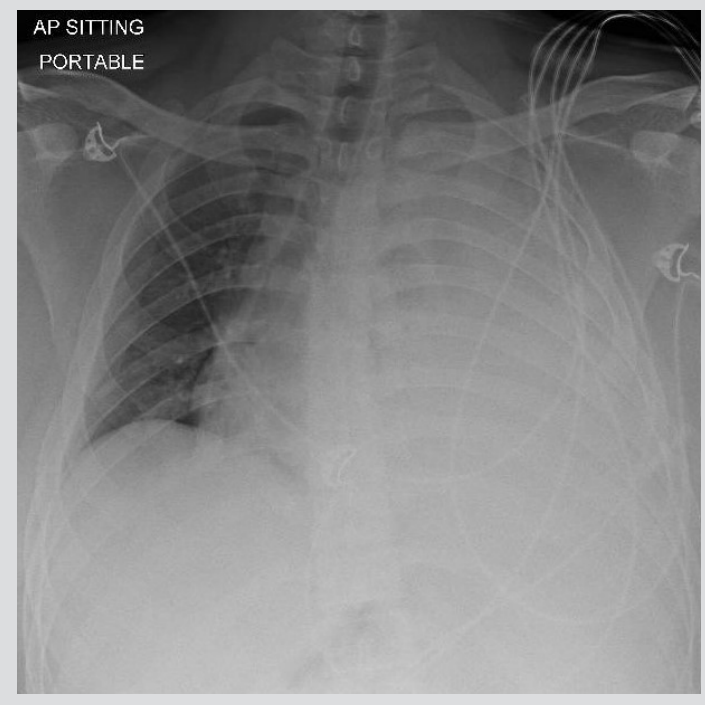

Figure 4. Chest $x$-ray showing a massive left pleural effusion with mediastinal shift 


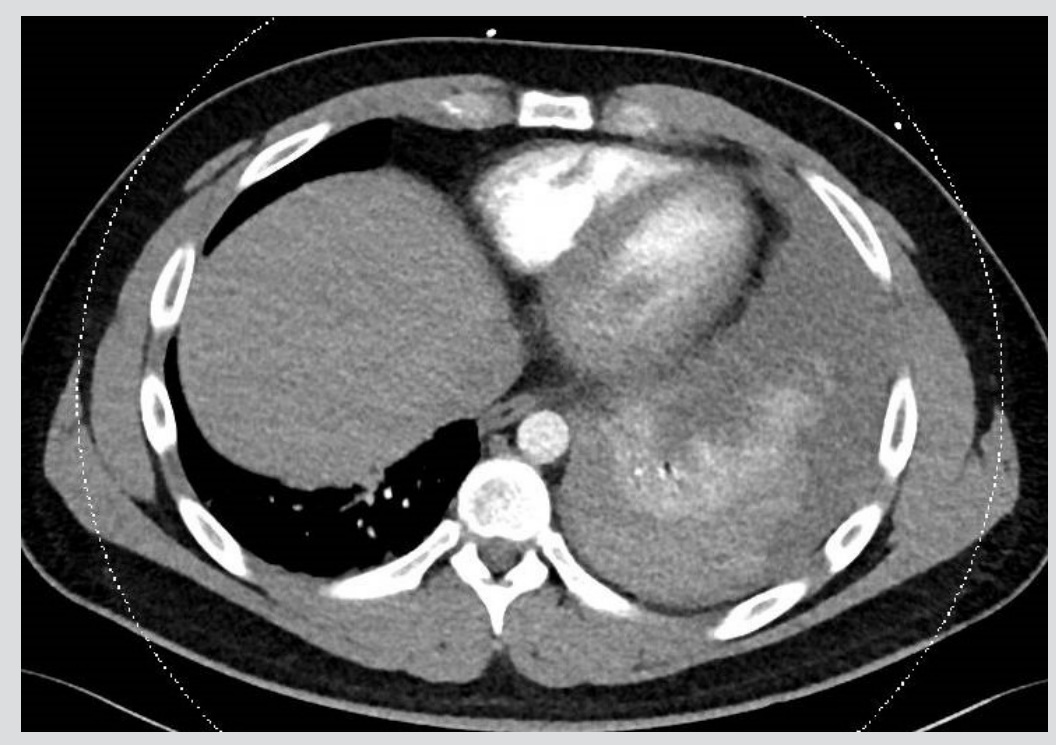

Figure 5. Computed tomography pulmonary angiogram showing a massive left haemothorax with collapse of the left lung

Rivaroxaban was stopped and four-factor prothrombin complex concentrate at $50 \mathrm{IU} / \mathrm{kg}$ was administered. A chest tube was inserted and immediately drained 2 litres of frank blood. The patient's haemoglobin dropped from a baseline of $14.0 \mathrm{~g} / \mathrm{dl}$ during the first admission to $7.4 \mathrm{~g} / \mathrm{dl}$. Video-assisted thoracoscopy was considered but not performed as there was no evidence of further bleeding. The chest tube was removed when there was no more drainage and the patient was followed up with repeat CXRs to ensure no further recurrence of the haemothorax.

\section{DISCUSSION}

This patient had pulmonary embolism with symptoms of severe pleuritis, but there was no obvious radiological evidence of pulmonary infarction on initial imaging. Pleuritic pain is a common symptom in patients with pulmonary embolism and associated pleural effusion ${ }^{[2]}$. As with this patient, they are managed according to standard of care and anticoagulation is not contraindicated ${ }^{[2]}$. Apart from clinical assessments, such pleural effusions are usually not monitored radiologically.

Pleural effusion secondary to pulmonary embolism may be further complicated by the development of haemothorax. This secondary event can occur 1-2 weeks after the patient starts anticoagulation ${ }^{[3,4]}$. This timing corresponds to a period of maximum tissue necrosis and breakdown after an infarction. The rupture of infarcted tissue and blood vessels near the visceral pleura can cause rapid accumulation of blood in the pleural cavity. Case reports of fatalities with post-mortem findings have demonstrated this phenomenon ${ }^{[4,5]}$.

Bleeding is further accentuated by the use of anticoagulants. Although this is the first report of spontaneous haemothorax with rivaroxaban, the few available publications mentioning other anticoagulants suggest it is not exclusive to any specific class of anticoagulant ${ }^{[5,6]}$.

This case highlights the fact that significant pleuritic pain in a patient with pulmonary embolism may indicate ischaemia or subclinical infarction of tissue near the visceral pleura with an increased risk of pleural effusion and the subsequent development of a haemothorax.

Such patients should be considered for closer monitoring and additional reassessment such as early repeat imaging. Especially since the increased use of direct oral anticoagulants has facilitated ambulatory care and shorter duration of hospitalisation, pulmonary embolism patients and their physicians should be more aware of this complication which may manifest in the outpatient setting. Patients should also be adequately warned of danger signals such as recurrence of pain or dyspnoea in the first 2 weeks after diagnosis.

\section{REFERENCES}

1. Patrini D, Panagiotopoulos N, Pararajasingham J, Gvinianidze L, Iqbal Y, Lawrence DR. Etiology and management of spontaneous haemothorax. J Thorac Dis 2015;7(3):520526.

2. Light RW. Pleural effusion in pulmonary embolism. Semin Respir Crit Care Med 2010;31(6):716-722.

3. Rostand RA, Feldman RL, Block ER. Massive hemothorax complicating heparin anticoagulation for pulmonary embolus. South Med J 1977;70(9):1128-1130.

4. Wick MR, Ritter JH, Schuller D. Ruptured pulmonary infarction: a rare, fatal complication of thromboembolic disease. Mayo Clin Proc 2000;75(6):639-642.

5. Simon HB, Daggett WM, DeSanctis RW. Hemothorax as a complication of anticoagulant therapy in the presence of pulmonary infarction. JAMA 1969;208(10):1830-1834.

6. Abu Hishmeh M, Srivastava P, Lougheide Q, Srinivasan M, Murthy S. Massive spontaneous hemothorax as a complication of apixaban treatment. Case Rep Pulmonol 2018;2018:8735036. 